\title{
Queen-produced volatiles change dynamically during reproductive swarming and are associated with changes in honey bee (Apis mellifera) worker behavior
}

\author{
Jessica Richards ${ }^{1}$, Morgan CARR-MarkelL ${ }^{2}$, Abraham HeFetz ${ }^{3}$, \\ Christina M. GrozingER ${ }^{1}$, Heather R. MATTILA ${ }^{4}$ \\ ${ }^{1}$ Department of Entomology, Center for Pollinator Research, Center for Chemical Ecology, 1 Chemical Ecology Lab, The \\ Pennsylvania State University, Orchard Road, University Park, PA 16802, USA \\ ${ }^{2}$ Department of Entomology, University of Minnesota, 219 Hodson Hall, 1980 Folwell Avenue, St. Paul, MN 55108, \\ USA \\ ${ }^{3}$ Department of Zoology, George S Wise Faculty of Life Sciences, Tel Aviv University, Ramat Aviv 69978, Israel \\ ${ }^{4}$ Department of Biological Sciences, Wellesley College, 106 Central Street, Wellesley, MA 02481, USA
}

Received 26 June 2014 - Revised 22 January 2015 - Accepted 16 February 2015

\begin{abstract}
During colony fission, honey bee workers are exquisitely sensitive to the presence of their queen in airborne swarms and bivouacs and will abandon swarming if she is absent. However, it is not known whether swarming queens produce a chemical bouquet that is distinct from non-swarming queens, containing either unique chemicals or altered proportions of chemicals. We found that queens emitted higher quantities and greater numbers of unique volatiles at liftoff than they did prior to swarming or in clustered bivouacs, and swarming workers tended to be attracted to these liftoff volatile blends. Pentadecane and heptadecane were collected most frequently and emitted in significantly higher quantities by queens at liftoff; these compounds have been described as pheromone components in other social insects, but not yet in honey bees. Our results suggest that volatile emission by queens is more dynamic than previously thought and that changes in their chemical signals may play a role in regulating the behavior of swarming workers.
\end{abstract}

honey bees / chemical ecology / pheromones / worker behavior / swarming

\section{INTRODUCTION}

Honey bee reproductive swarming is a fascinating example of a collective behavior for which the movement of thousands of individuals must be finely coordinated (reviewed by Seeley 2010 and Grozinger et al. 2014). During reproductive swarming, approximately three quarters of

Electronic supplementary material The online version of this article (doi:10.1007/s13592-015-0358-x) contains supplementary material, which is available to authorized users.

Corresponding author: H. Mattila,

hmattila@wellesley.edu

Manuscript editor: David Tarpy workers and their queen leave the nest and form a temporary cluster (called a bivouac) from which they search for nest sites and eventually relocate en masse to their most popular selection (Rangel and Seeley 2012; Rangel et al. 2013). Several studies have elucidated the behavioral signals that are produced by workers to regulate the swarming process (reviewed by Seeley 2010 and Grozinger et al. 2014). However, queens also produce pheromonal signals that are known to trigger the initiation of new queen rearing in preparation for swarming (Fefferman and Starks 2006; reviewed in Grozinger et al. 2014) and to keep flying and clustered swarms cohesive (Butler et al. 1964; Butler and Simpson 1967; Avitabile et al. 1975; Winston et al. 1982). Although it is not known 
whether queens play an active role in coordinating swarming, workers in a swarm will not form a bivouac or relocate to a new nest site unless their queen joins them (Avitabile et al. 1975; Pierce et al. 2007), which suggests that queen-derived signals might help to coordinate these group transitions. In this study, we investigated the pheromone profiles of swarming queens, and the response of workers to volatiles from swarming and non-swarming queens, to determine whether dynamic changes in queen volatiles occur as queens swarm and whether such changes are linked to the behavior of swarming workers.

Pheromones produced by queens have been shown to play a role in regulating the initiation of queen rearing, which is the first step in swarm preparation. Queens deposit footprint pheromones (the composition of which has not yet been determined) from their tarsal glands as they walk, which inhibit new queen rearing in congested colonies that would typically swarm (Lensky and Slabezki 1981). New queen rearing in congested colonies is also inhibited by queen mandibular pheromone, or QMP, which is produced by the mandibular glands (Slessor et al. 1988, 1990; Winston et al. 1991). QMP consists of 9-oxo-( $E)$-2-decenoic acid (9-ODA), $(R)$ - and $(S)$-9-hydroxy-( $E)$-2-decenoic acid (9-HDA), methyl $p$-hydroxybenzoate (HOB), and 4hydroxy-3-methyoxyphenylethanol (HVA) (Slessor et al. 1988). Approximately 20 other chemical components are found in the mandibular glands, and the proportions of these components change with reproductive state and mating quality, which has an influence on worker physiology and behavior (Richard et al. 2007, 2011; Kocher et al. 2009; Niño et al. 2012, 2013; Peso et al. 2013).

The presence of queens or their pheromones also improves swarm cohesion after workers leave their parental nest, both when swarms are airborne and when they are clustered. Airborne swarms span larger areas if they are queenless (Morse 1963; Beekman et al. 2006). Swarming workers will also return to their queen if she is prevented from flying with them, and will readily relocate if the queen is moved short distances from the bivouac (Avitabile et al. 1975; Morse 1963; Simpson 1963). Interestingly, a queenless swarm will not return to the parental hive or will lose cohesion if a swarming worker is marked with 9-ODA, which suggests that queens signal their presence in part with this main component of QMP (Avitabile et al. 1975). Similarly, swarms are attracted to, and cluster stably around, lures impregnated with 9-ODA, 9-HDA, and QMP (Butler et al. 1964; Butler and Simpson 1967; Winston et al. 1982). However, live queens or whole-queen extracts attract and stabilize swarms more effectively than lures (Boch et al. 1975; Winston et al. 1989), thus additional pheromone components are likely involved in cluster cohesion.

It is unknown whether queens simply passively signal their presence to the swarming workers via their standard pheromone blend, or whether their pheromone blend changes in composition (in either compound type or quantity) during swarming. Equivalent levels of 9-ODA are produced by swarming and non-swarming queens (Seeley and Fell 1981), but other pheromone components have not been examined. Because the relative proportions of the non-QMP components of the mandibular gland change significantly with queen reproductive state and mating quality, similar changes in the pheromone bouquet of queens between stages of swarming could improve bivouac cohesion, signal the queen's presence to airborne swarms, or modulate worker behavior in swarms. There is some evidence that queenworker interactions change during swarming: although older workers (foragers and scouts) are not attracted to queen pheromones (Grozinger and Robinson 2007) and do not interact with queens in non-swarming, established colonies (Seeley 1982), scouts tend to seek out queens in swarm bivouacs (Pierce et al. 2007).

To evaluate whether queen pheromone blends change dynamically during swarming, we used solid-phase microextraction (SPME) coupled with gas chromatography/mass spectroscopy (GC/MS) to non-destructively sample and characterize the volatile chemical blends produced by queens at three different stages of the swarming process: in colonies prior to the initiation of swarming, in clustering bivouacs before house hunting begins, and during bivouac liftoff. We focused on volatile blends because the behavioral changes that occur at swarm liftoff are rapid and are less likely to be associated with changes in contact pheromones, 
given that the queen must communicate with the swarm while airborne. We compared the volatile profiles of swarming and non-swarming queens to determine (1) whether the quantities of volatiles that are released by queens change as they go through different stages of the swarming process, and (2) whether queens release different types of volatiles during swarming. Finally, to determine whether worker behavior differs in response to the chemical blends produced by queens at different stages of the swarming process, we assayed the relative attraction of swarming workers to the chemicals collected from swarming queens at liftoff versus those collected from queens in nonswarming colonies.

\section{MATERIALS AND METHODS}

\subsection{Honey bee colonies}

Colonies of honey bees (Apis mellifera L.) were maintained according to standard beekeeping practices at the Wellesley College apiary in Wellesley, MA, USA. Naturally mated honey bee queens and colonies were purchased in 2010 from Merrimack Valley Apiaries (Billerica, MA, USA) and in 2012 from Beehavin Apiaries (Smithfield, RI, USA). Queens were primarily of mixed Carniolan (2010) or Italian (2012) descent and were reared in the year that they were studied. Colonies were established from five-frame nucleus colonies in the spring of each year and had expanded in size to fill two Langstroth hive bodies when swarms were assembled. Samples of queen volatiles were collected between 22 June and 2 September 2010. Behavioral assays were performed between 6 July and 27 July 2012.

\subsection{Swarm establishment}

Bivouacking "swarms" were created using standard methods (Seeley and Buhrman 1999). Briefly, approximately $1-1.5 \mathrm{~kg}$ of workers (7,500-11,000 workers; Mitchell 1970) from a single colony were shaken off frames of comb and into a wooden box with wire-screen sides $(15 \times 25 \times 35 \mathrm{~cm})$; the colony's queen was placed in an unused plastic queen cage ( $24 \mathrm{~h}$ prior to swarm creation), which was secured inside the box so that workers could cluster around it in a bivouac. Once in the screened box, each cluster was fed a 1:1 sugar/water solution ad libitum for at least $6 \mathrm{~h}$ per day for 2 to 3 days to simulate engorgement by workers on honey, as would occur prior to a natural swarm exodus (Combs 1972). After this feeding period was over, the caged queen was removed from the screened box and hung on the board of a swarm stand (Supplementary Figure S1a). Workers were shaken out of the box and onto the base of the stand and then moved to the board to resettle in a bivouac around the queen. At this point, the swarm began its search for a new nest site and was subsequently monitored for preparation for liftoff by watching waggle dances and listening for worker piping, signals that change characteristically as house hunting progresses toward liftoff (reviewed in Seeley 2010).

\subsection{Sampling queen volatile profiles}

Volatile samples were collected from caged queens at three stages of swarming for each colony a queen headed. Each queen was sampled at each time point. "In-hive" samples were collected $24 \mathrm{~h}$ after each queen was caged, but before a swarm was shaken into its screened box, so the queen was still in her hive. "Cluster" samples were collected after the ad libitum feeding period was over and immediately before colonies were transferred to swarm stands, so queens and workers had been in a homeless cluster for some time but had not yet begun the house-hunting process. "Liftoff" samples were collected immediately after bivouacs dissolved and airborne swarms began to move to their new home. A total of 17 queens were sampled at all three stages of swarming and were considered in the analysis.

To monitor the volatiles that were produced by the same set of queens across these time points, we used solid-phase microextraction (SPME) coupled with gas chromatography/mass spectroscopy (GC/MS). SPME has been used previously to characterize volatile chemical profiles of honey bee queens (Gilley et al. 2006; Huang et al. 2009) and waggle-dancing honey bee workers (Thom et al. 2007). We used $65-\mu \mathrm{m}$ polydimethylsiloxane divinylbenzene SPME fibers (Supelco SPME fiber 57326U; Sigma-Aldrich, Bellefonte, PA, USA) because they capture the largest number of queen-produced volatiles compared to other fiber types (Gilley et al. 2006; Huang et al. 2009). Fibers were decontaminated before each exposure to a queen by insertion into a GC/MS at $250{ }^{\circ} \mathrm{C}$ for $3 \mathrm{~min}$, and then the end of the fiber assembly was wrapped with Teflon tape to minimize adsorption of environmental volatiles prior to exposure to queens. Fibers were then inserted 
for 15 min into collection chambers with queens (see Supplementary Figure S1b). The ends of the fiber assemblies were rewrapped with Teflon tape after exposure to queens and prior to desorption.

Before sampling a queen, each collection jar was sampled empty to generate its "blank" volatile profile (in the field and on the same day that it was used to sample a queen). Blank and queen samples were collected from jars for 15 min under ambient field conditions and in a shaded area of the apiary. Different fibers were used for each collection. Fibers were inserted into the GC/MS as soon as possible and always on the same day that volatiles were collected.

We also performed a series of controls to determine whether the volatile profiles obtained from swarming workers were similar to those obtained from queens (Supplementary Figures S2 and S3). First, we sampled the volatiles produced by a bivouac by inserting a caged SPME fiber into a bivouac for $30 \mathrm{~min}$ (see Supplementary Figure S3 for the chromatogram). Next, we repeatedly rubbed a SPME fiber across the abdomen of two buzz-running workers minutes before swarm liftoff (Supplementary Figure S3).

\subsection{Analyzing queen volatile profiles}

Volatiles from blank and queen samples were analyzed by inserting and desorbing SPME fibers into a 5890 Series II Plus Gas Chromatograph in splitless mode equipped with an HP-5MS capillary column (5\% phenyl-95\% methylsiloxane; 30-m length and 0.25-mm inner diameter; Hewlett-Packard Company, Palo Alto, CA, USA). Helium was used as the carrier gas at a pressure of $12 \mathrm{psi}$ (flow rate $=1.0 \mathrm{~mL} / \mathrm{min}$, velocity $=37.4 \mathrm{~cm} / \mathrm{s}$ ). The oven temperature was held at $40{ }^{\circ} \mathrm{C}$ for $3 \mathrm{~min}$ before being raised at a rate of $15^{\circ} \mathrm{C} / \mathrm{min}$ to $250{ }^{\circ} \mathrm{C}$; the injector temperature was set at $240{ }^{\circ} \mathrm{C}$. The $\mathrm{GC}$ was equipped with a Hewlett Packard 5972 Mass Selective Detector (HewlettPackard Company, Palo Alto, CA, USA), operated in EI mode at $150 \mathrm{eV}$ according to Gilley et al. (2006). Compound identification was deduced from the pattern of mass fragmentation as compared to the National Institute of Standards and Technology (NIST) mass spectrometry library. Quantitative analyses were performed from the Total Ion Current (TIC) data using ChemStation software (Agilent Technologies, Inc., Santa Clara, CA, USA; see Supplementary Figure S2 for sample chromatograms). Peak integration was performed automatically by using the "Auto Integrate" function of the ChemStation software. Any compounds that were present in the blanks were removed from the analysis of that sample. The total number of compounds (peaks) produced per queen and the total quantity produced per queen (calculated as the total area under all peaks) were determined. Note that we did not use an internal standard because of the potential risk of its peak obscuring volatile peaks. Thus, our chemical analyses favored better information about total number of compounds over compound quantity.

Given natural variation among queens in volatile production (see Supplementary Figure S4), we next identified regularly occurring "candidate compounds," defined as compounds that were produce by at least six, or approximately one third, of queens within a single time point. Selecting this threshold gave us manageable and sufficient number of compounds (eight) to use for an analysis; if we had used compounds present in one half of the queens at a single time point (i.e., 9 of the queens, since 17 queens were sampled at liftoff), we would have had only two candidate compounds to consider ( $(E / Z)$ - $\beta$-ocimene and pentadecane). Each compound was located in the chromatograms according to its retention time (within a window of $\pm 0.02 \mathrm{~min}$ across samples) and its mass spectrum. For seven of these candidate compounds, the fragmentation patterns in their mass spectra matched exactly with known compounds in the NIST database, allowing us to identify them conclusively (see Table I).

\subsection{Assessing attraction of workers to queen volatiles}

We conducted a choice-test assay to determine whether swarming workers were more attracted to the volatiles produced by swarming queens or nonswarming queens. To do this, bivouacking swarms were created from eight colonies using the above methods and then monitored for liftoff. Each swarming queen was paired at random with a non-swarming queen (control) from a colony that was established in the same year and from the same commercial stock (see Sect. 2.1; these were different colonies than those used for sampling queen profiles, Sect. 2.3). Non-swarming queens came from source colonies that were actively rearing brood and showed no signs of queen rearing. Each queen was placed in a new plastic cage the day that her swarm counterpart was moved to a swarm stand 


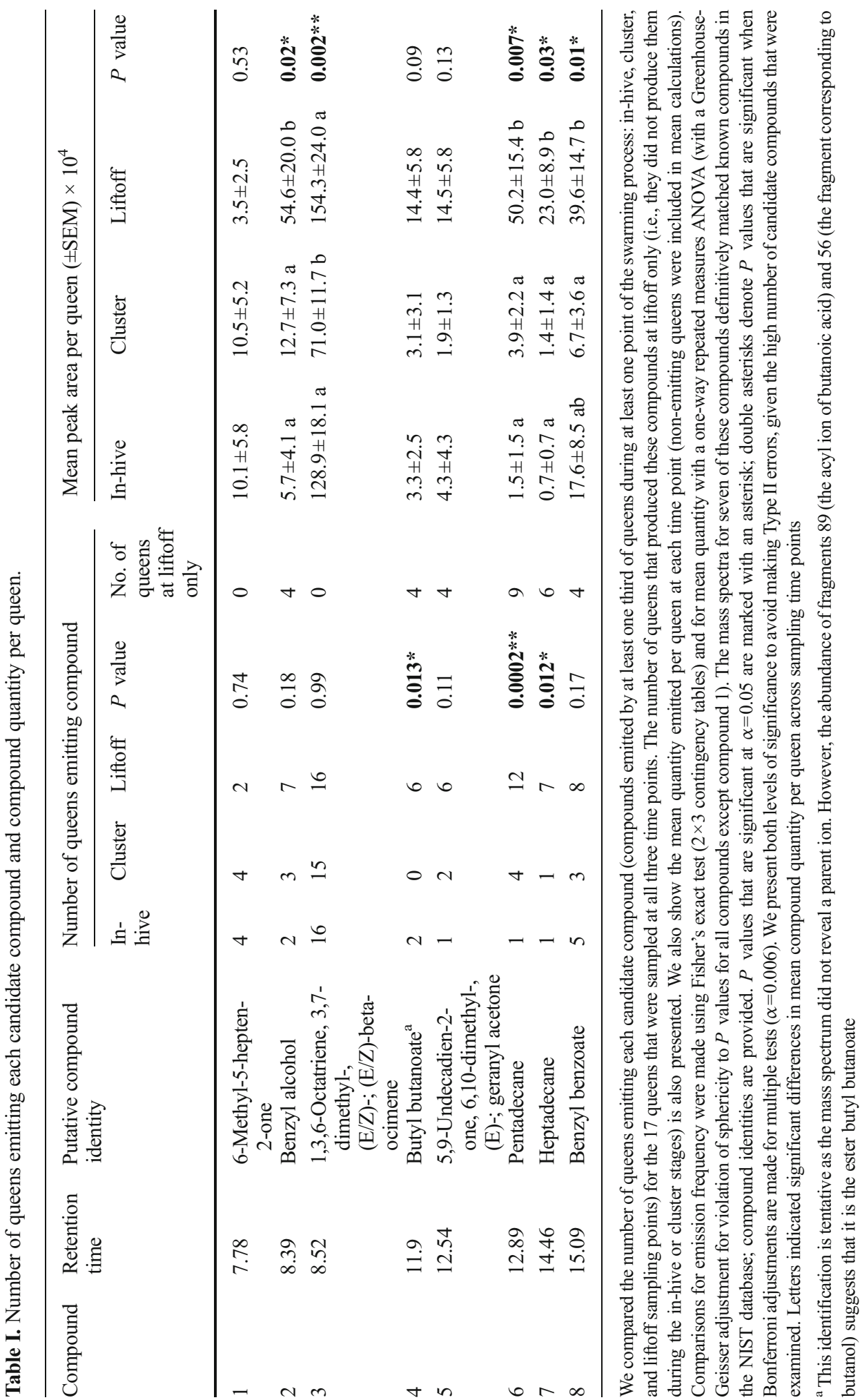


(approximately 1-2 days prior to liftoff); the cage was kept in the hive's brood area until the other queen's swarm lifted off. When a bivouac lifted off, its queen and her non-swarming counterpart were placed in separate collection jars for $15 \mathrm{~min}$ (see Supplementary Fig S1b for detailed information about collection jars).

Using an insect vacuum (BioQuip, Rancho Dominguez, CA, USA), approximately 300-400 workers were taken from a bivouacking swarm that was house hunting (a different swarm than the one that had the swarming queen to be tested) and the workers were introduced into a choice-test apparatus (Supplementary Figure S5). A new apparatus was used for each replicate. Once test workers were in the apparatus, they were placed in a shaded area and covered with a dark cloth for at least $30 \mathrm{~min}$ to give them time to acclimate to the testing arena.

To start the behavioral assay, tightly fitting pieces of Tygon tubing were inserted into the mouths of the collection jars, and the other end of the tubes were firmly attached to the holes on the sides of the choicetest box. The box and attached pair of jars were then covered with a dark cloth and workers were permitted to move freely about the box and into either jar. After $1 \mathrm{~h}$, the jars were removed, sealed, and chilled, and then the number of workers in each jar was counted. This trial was repeated eight times with eight unique pairs of swarming and non-swarming queens (queens were used only once in each category and swarms were used only once as a source of test workers).

\subsection{Statistical analyses}

Mean number, total quantity of compounds, and quantity of individual compounds produced by queens were analyzed by one-way repeated measures ANOVA to determine whether values changed over the three sampling points (within-subject effect; a Greenhouse-Geisser $P$ value adjustment was used when Mauchley's test showed that the assumption of sphericity was not met). We conducted posttest mean contrasts when within-subject effects were significant (using Bonferroni corrections to adjust for multiple comparisons). Once we identified candidate compounds (see above), we compared the number of queens that produced each compound across the three stages of swarming with a Freeman-Halton extension of a Fisher exact probability test for a $2 \times 3$ contingency table. We compared the mean number of workers attracted to jars containing the volatiles of swarming versus non-swarming queens with a paired $t$ test (the data were normal, despite a small sample size; ShapiroWilks test, $\mathrm{W}=0.93, P=0.52$ ). All statistical analyses were performed using SAS 9.3 (SAS Institute Inc., Cary, NC, USA), with the exception of the post-test contrasts and the $2 \times 3$ contingency tables, which were performed using open-access statistical calculators (graphpad.com/quickcalcs/posttest1.cfm and vassarstats.net/fisher $2 \times 3 . h t m l$, respectively). Data are reported as mean \pm SEM.

\section{RESULTS}

\subsection{Volatile profiles of swarming queens}

Queens emitted a distinct profile of compounds relative to bivouacking workers or buzz-running workers and, in general, greater quantities of volatiles were collected from queens (see Supplementary Figures S2 and S3 for sample chromatograms). Queens emitted significantly greater numbers of compounds at liftoff than in hives or in clustered bivouacs (Figure 1;

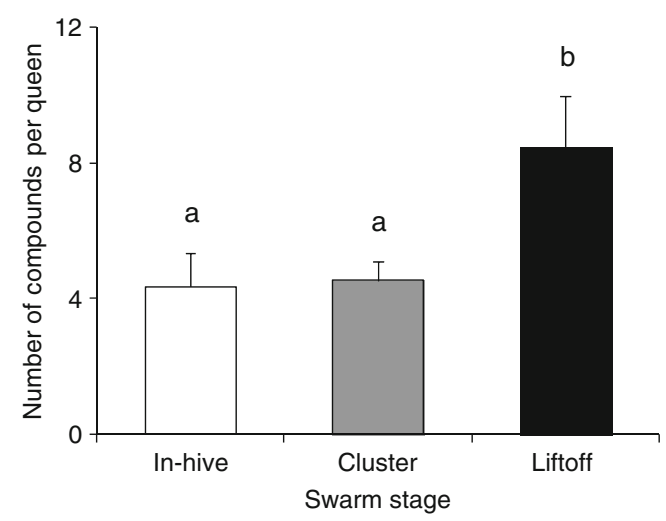

Figure 1. Total number of compounds emitted by queens. The number of volatile compounds emitted per queen during stages of swarming was determined using gas chromatography (see Sect. 2). Queens emitted significantly more compounds at liftoff than they did before they swarmed (in-hive samples) or in clusters before house hunting (cluster samples) (repeated measures ANOVA, $\mathrm{F}_{(2,32)}=5.2, P=0.01 ; n=17$ queens). Sample points with different letters have significantly different means. 
repeated measures ANOVA, $\mathrm{F}_{2,32}=5.2, P=0.01$ ). Furthermore, the total quantity of volatiles emitted by queens increased as swarming progressed, with the lowest levels collected from queens that had not yet begun the swarming process, intermediate levels from queens in clusters prior to house hunting, and the highest levels from queens that were trying to lift off with swarms (Figure 2; repeated measures ANOVA: $\left.F_{2,32}=5.4, P=0.009\right)$. Note that we did not use an internal standard, which makes it difficult to precisely measure total quantities of compounds that were produced. However, differences among stages of swarming represent broad relative changes in volatile emission across these groups of queens.

In total, 84 unique compounds (not found in the blanks) were present across all the queen-derived samples. However, the number of compounds that were emitted by each queen varied considerably (Supplementary Figure S4), with queens emitting between $0-15$ compounds when they were in hives, 2-10 compounds when they were clustered around the queen, and 1-24 compounds at the moment of liftoff. To reduce the complexity of the data set and focus on compounds that were present most commonly in these samples, we

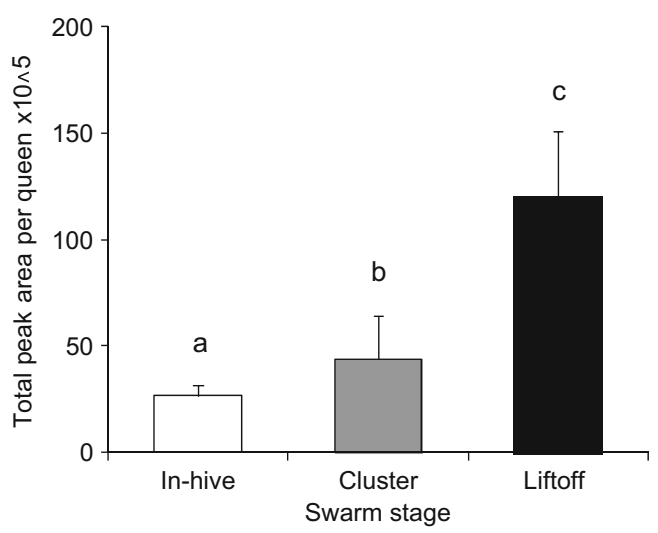

Figure 2. Total quantity of volatiles emitted by queens. The total quantity of volatiles emitted per queen during stages of swarming was determined using gas chromatography (see Sect. 2). The quantities of volatiles emitted by the queen changed significantly as swarming progressed (repeated measures ANOVA, $\mathrm{F}_{(2,32)}=5.4$, $P=0.01 ; n=17$ queens). Sample points with different letters have significantly different means. screened for compounds that were emitted by at least six queens in a single phase of swarming (approximately one third of queens in a sample group). Based on these criteria, we selected eight "candidate compounds" for additional analysis (Table I). Although this was a relatively small number of compounds, they contributed substantially to the total chemical output of the queens. For the 51 samples/chromatograms that were analyzed for this study, these compounds accounted for $75-100 \%$ of the total volatile quantity produced in 17 of them, $50-75 \%$ in 18 of them, and less than $50 \%$ in 16 of them.

We found that three of the eight candidate compounds showed a significant change in the number of queens that emitted them over the sampling time points, with most being emitted almost exclusively by queens at liftoff: These were compounds 4, 6, and 7 (Table I). No difference across time points in the number of queens that emitted the remaining five candidate compounds could be detected. When the quantity of individual compounds that were emitted by queens was examined at each time point, emission of five of the eight compounds increased significantly toward liftoff (compounds 2, 3, 6, 7, and 8; Table I). Only compounds 6 and 7, pentadecane and heptadecane, increased in both the number of emitting queens and quantity emitted per queen as swarming progressed (Table I). Notably, pentadecane was found in a large fraction (12 out of 17) of the liftoff queens.

\subsection{Attraction of workers to queen volatiles}

Of the workers that made a choice in the choice test, an average of $71 \pm 12 \%$ versus $29 \pm 12 \%$ were attracted to volatiles produced by swarming queens compared to non-swarming queens (Supplementary Table SI). However, our sample size was small and the difference in mean number of workers choosing swarming versus nonswarming queens only approached significance (paired $t$ test; $\mathrm{t}_{(7)}=1.8, P=0.12$ ). More workers were attracted to swarm queen volatiles in five of eight trials compared to non-swarming queen volatiles in two of eight trials, with no clear outcome in the last trial (Supplementary Table SI). 


\section{DISCUSSION}

Overall, our results indicate that volatile emission by queens changes at swarm liftoff. By sampling the total airspace volatiles that are emitted by queens as they transition with workers from house hunting toward liftoff, we demonstrated that queens emit a significantly greater number of volatile compounds when bivouacs lift off than when they are clustered around the queen prior to house hunting or in the natal nest before swarming begins. The total quantity of these compounds also increased as queens approached swarm liftoff, with liftoff queens emitting significantly more volatiles than in-hive or bivouacking queens. We identified two compounds (heptadecane and pentadecane) that were produced by significantly more queens at liftoff and whose quantities were also significantly higher at liftoff. While previous studies of changes in pheromone production by queens have focused on relatively stable long-term differences between virgin and mated queens (Slessor et al. 1990; Gilley et al. 2006) or among mated queens with different mating qualities (Huang et al. 2009; Niño et al. 2013), our results suggest that mated queens can alter their chemical output in response to relatively short-term changes in the state of the social collective, as workers do when they release Nasonov or alarm pheromones in response to quickly shifting conditions (Pickett et al. 1980; Breed et al. 2004). These results suggest that queens may play a more active role in organizing the swarming process than was previously known. Given that swarming workers tended to be more attracted to the volatiles from queens at liftoff compared to those of non-swarming queens, it seems more likely that these signals serve to keep airborne workers informed that a queen is traveling with them than to help dissolve bivouacs as they take flight, but we cannot rule out either possibility.

There was considerable variation among queens in both total volatile output and in the total number of compounds that they emitted. Honey bee workers are capable of distinguishing their own queen from others and newly introduced queens are often executed (Breed 1981), so this variation is likely associated in part with kin recognition cues. There was no single compound that was emitted by all swarming queens at liftoff, which suggests that it is a blend of compounds (or perhaps their relative proportions) rather than a single compound that elicits behaviorally relevant worker responses. The use of blends of compounds that vary across individuals within a species is common in chemical communication systems (Wyatt 2010). It is also possible that additional active compounds may have been emitted that were not efficiently captured using our methods because it is unlikely that a single type of SPME fiber adsorbs $100 \%$ of the volatiles that are emitted by queens.

Two compounds (the hydrocarbons pentadecane and heptadecane) were emitted by significantly more queens during swarm liftoff than when the same queens were in colonies or in clusters prior to liftoff, and were emitted at significantly higher quantities by liftoff queens, making these compounds excellent candidates for investigation as novel pheromones that regulate the behavior of workers at the moment of liftoff and when swarms are airborne. Notably, pentadecane was also found in a relatively large number of liftoff queens (12 out of 17 queens, see Table I). Both pentadecane and heptadecane are found in trace quantities in honey bee queen Dufour's glands and on the surface of queen-laid eggs, and pentadecane is also found on workerlaid eggs (Katzav-Gozansky et al. 2003; Richard et al. 2011). Neither of these compounds has been identified as a component of honey bee Nasanov gland pheromone, which is released by workers to attract other workers during swarming, though hydrocarbons were not specifically investigated in these studies (Pickett et al. 1980). These compounds have also been found in trace quantities in the sting apparatus of honey bee workers (McDaniel et al. 1984), but are not considered components of alarm pheromone, which elicits worker attraction (Breed et al. 2004). In contrast, these compounds are present in large quantities in Dufour's and venom glands of many other social insect species, for which they appear to function in worker attraction. Both pentadecane and heptadecane are found in the Dufour's gland of Myrmica ants, and gland extracts are attractive to workers (Cammaerts et al. 1981). Similarly, both compounds are found in the venom glands of Polybioides wasps (pentadecane is a major 
component), and extracts of these glands also trigger worker attraction (Sledge et al. 1999). Finally, pentadecane is a volatile component of alarm pheromone of Camponotus ants, though paradoxically, it is found to calm worker ants when presented on its own (Fujiwara-Tsujii et al. 2006). Thus, it is possible that these compounds are produced by another gland of honey bee queens or are produced by the Dufour's glands of swarming honey bee queens only, and may function in worker attraction or swarm cohesion.

The remaining candidate compounds were produced either by equivalent numbers of queens or in equivalent quantities across the three stages of swarming that we tested. Butyl butanoate was produced by significantly more liftoff queens, but levels per queen were not significantly higher (Table I). Although esters are quite common in bee exocrine glands (Francke et al. 1984), butyl butanoate has not yet been reported as a beeproduced compound. However, it is interesting that this compound, in conjunction with 2-methyl-1butanol, has been reported as a primer pheromone that inhibits the production of secondary queens in the termite Reticulitermes speratus (Matsuura et al. 2010). ( $E / Z)$ - $\beta$-ocimene was produced by queens at all stages, but levels of this compound were significantly higher in liftoff queens (Table I). $(E)$ - $\beta$-ocimene was previously identified as a queen volatile that is emitted at higher levels by egg-laying queens than by unmated queens (Gilley et al. 2006; Huang et al. 2009). Benzyl alcohol, geranyl acetone, and benzyl benzoate trended toward being emitted by more liftoff queens than inhive queens, and benzyl alcohol and benzyl benzoate and were produced at significantly higher quantities in liftoff queens (Table I). Geranyl acetone is likely a biosynthetic derivative of farnesol (Schulz et al. 2011), which is a component of worker Nasanov glands (Pickett et al. 1980). The five-compound Nasanov pheromone blend is attractive to honey bee swarms (Schmidt 1999).

It is important to note that workers could also be releasing behaviorally active compounds in bivouacs and during swarm liftoff. It is possible that these compounds could have been released in sufficient quantities that they "contaminated" the queen, and thus, our samples of queen volatiles may have included worker-produced chemicals, and worker responses in our behavioral bioassay may reflect in part responses to these chemicals. It seems unlikely that workers produced sufficient quantities of compounds that they contaminated caged queens: We did not detect similar chemicals in our control collections from swarming workers (see Supplementary Figure S3) and, overall, the numbers and quantities of chemicals obtained from the worker samples were substantially lower than those obtained from the queens. It is possible that during the choice test, workers signaled to each other and thus the first workers to make a choice to enter a volatile-laden jar influenced the response of the other workers. However, these potential secondary signals would simply amplify an existing worker preference, rather than create that preference. In five of the eight trials, workers preferred the chamber that contained the swarming queen, while in only two trials was there a preference for the non-swarming queen. Although there is variability in queens' chemical signals and workers' responses to these signals, these data suggest that, in general, the chemicals produced by swarming queens are an important part of a potential suite of signals that attract workers to their queen during swarm liftoff.

Although all five components of QMP were detectable by our SPME sampling method (data not shown), none of them were detected from the queens in our study nor were they reported by other studies that used SPME to sample queen volatiles (Gilley et al. 2006; Huang et al. 2009). Previous studies suggest that 9-ODA is important for stabilizing swarms (Butler et al. 1964) and for worker detection of the presence of queens in airborne swarms (Avitabile et al. 1975), while HVA also helps to stabilize and attract swarms (Winston et al. 1982). Thus, while QMP components may play a role in swarm liftoff, these chemicals may not have been volatized in high enough concentrations to be readily detected by our methods. Interestingly, QMP is typically considered to be a pheromone that is transmitted among workers by contact (Seeley 1979; Naumann et al. 1993), but workers in airborne swarms and drones during mating flights can clearly detect 9-ODA (Avitabile et al. 1975; Brockmann et al. 2006). It is possible that queen pheromones that function as contact pheromones 
to mediate queen-worker interactions within colonies are coupled with additional volatiles to mediate long-range queen-worker and queen-drone interactions during flight.

Swarming is a fascinating example of collective behavior. While previous studies have focused primarily on the role that workers play in initiating swarm preparations, stimulating liftoff from the established colony and the bivouac, and scouting for new nest sites (reviewed in Seeley 2010 and Grozinger et al. 2014), our results demonstrate that queens are not simply passive participants in this process. Although a queen is unaware of her swarm's destination and likely has little influence over the speed of the househunting process, it is critical that she accompany workers each step of the way or the swarming process will fail. Accordingly, workers need reliable information from their queen about her presence in a swarm, and undoubtedly there is strong selection on queens to produce such signals. Our results provide the first evidence that the volatile blends that are emitted by queens change as bivouacs prepare to lift off and that workers can respond behaviorally to these changes. Thus, both the individual (the queen) and the group (the swarming workers) play a role in this collective behavior. Further studies are necessary to determine the source of the variation in queen volatile profiles, to test the biological activity of pentadecane and heptadecane during swarm liftoff, to identify the stimuli that trigger these pheromone blend changes, to characterize the proximate mechanisms that underlie these changes, and to determine whether altered blends serve simply to confirm the presence of the queen or to actively influence the behavior of swarms.

\section{ACKNOWLEDGMENTS}

We thank Victoria Ellis, Elizabeth Brown, Michael Smith, and Katie McDonald for their assistance in the field; Elaine Igo for assistance with GC/MS configuration; Jim Tumlinson for access to the ChemStation software and the NIST mass spectroscopy library; and Bernardo Niño for his help in designing and building the behavioral assay apparatuses. We also thank Elina Lastro Niño and Etya Amsalem for critical reading of the manuscript. This study was funded by an NSF
CAREER and USDA-AFRI (2009-05207) grant to CMG, a BSF grant to AH and CMG, and an HHMI Postgraduate Internship, a Brachman-Hoffman grant, and a funds from a Knafel Endowed Chair in the Natural Sciences (Wellesley College) to HRM.

Les substances volatiles émises par la reine sont modifiées de façon dynamique durant l'essaimage reproductif et sont associées à des modifications du comportement chez les ouvrières d'abeilles (Apis mellifera )

écologie chimique / phéromone / comportement des ouvrières / essaimage

Von Königinnen produzierte Duftstoffe verändern sich dynamisch während des Schwarmvorganges und gehen einher mit Veränderungen im Verhalten von Bienenarbeiterinnen (Apis mellifera)

Honigbienen / chemische Ökologie / Pheromone / Arbeiterinnenverhalten / Schwärmen

\section{REFERENCES}

Avitabile, A., Morse, R.A., Boch, R. (1975) Swarming honey bees guided by pheromones. Ann. Entomol. Soc. Am. 68, 1079-1082

Beekman, M., Fathke, R.L., Seeley, T.D. (2006) How does an informed minority of scouts guide a honeybee swarm as it flies to its new home? Anim. Behav. 71, 161-171

Boch, R., Shearer, D.A., Young, J.C. (1975) Honey bee pheromones: field tests of natural and artificial queen substance. J. Chem. Ecol. 1 (1), 133-148

Breed, M.D. (1981) Individual recognition and learning of queen odors by worker honeybees. P. Natl. Acad. Sci. USA 78(4), 2635-2637

Breed, M.D., Guzmán-Novoa, E., Hunt, G.J. (2004) Defensive behavior of honey bees: organization, genetics, and comparisons with other bees. Annu. Rev. Entomol. 49, 271-298

Brockmann, A., Dietz, D., Spaethe, J., Tautz, J. (2006) Beyond 9-ODA: sex pheromone communication in the European honey bee Apis mellifera L. J. Chem. Ecol. 32 (3), 657-667

Butler, C.G., Simpson, J. (1967) Pheromones of the queen honeybee (Apis mellifera L.) which enable her workers to follow her when swarming. Proc. R. Entomol. Soc. A. 42 (10-12), 149-154

Butler, C.G., Callow, R.K., Chapman, J.R. (1964) 9hydroxydec-trans-2-enoic acid, a pheromone stabilizing honeybee swarms. Nature 201 (4920), 733 
Cammaerts, M.C., Evershed, R.P., Morgan, E.D. (1981) Comparative study of the dufour gland secretions of workers of four species of Myrmica ants. J. Insect. Physiol. 27 (1), 59-65

Combs, G.F. (1972) The engorgement of swarming worker honeybees. J. Apic. Res. 11, 121-128

Fefferman, N.H., Starks, P.T. (2006) A modeling approach to swarming in honey bees (Apis mellifera). Insectes. Soc. $53(1), 37-45$

Francke, W., Schröder, W., Bergström, G., Tengö, J. (1984) Esters in the volatile secretion of bees. Nova. Acta. Regiae. Societatis. Scientiarum. Upsaliensis. Serie. C. 3, 127-136

Fujiwara-Tsujii, N., Yamagata, N., Takeda, T., Mizunami, M., Yamaoka, R. (2006) Behavioral responses to the alarm pheromone of the ant Camponotus obscuripes (Hymenoptera: Formicidae). Zool. Sci. 23 (4), 353-358

Gilley, D.C., DeGrandi-Hoffman, G., Hooper, J.E. (2006) Volatile compounds emitted by live European honey bee (Apis mellifera) queens. J. Insect. Physiol. 2, 520527

Grozinger, C.M., Robinson, G.E. (2007) Endocrine modulation of a pheromone responsive gene in the honey bee brain. J. Comp. Bio. A. 193 (4), 461-470

Grozinger, C.M., Richards, J., Mattila, H.R. (2014) From molecules to societies: mechanisms regulating swarming behavior in honey bees (Apis spp.). Apidologie. 45 (3), 327-346

Huang, M.H., DeGrandi-Hoffman, G., LeBlanc, B. (2009) Comparisons of the queen volatile compounds of instrumentally inseminated versus naturally mated honey bee (Apis mellifera) queens. Apidologie. 40 (4), $464-471$

Katzav-Gozansky, T., Soroker, V., Kamer, J., Schulz, C.M., Francke, W., Hefetz, A. (2003) Ultrastructural and chemical characterization of egg surface of honeybee worker and queen-laid eggs. Chemoecology. 13 (3), 129-134

Kocher, S.D., Richard, F.J., Tarpy, D.R., Grozinger, C.M. (2009) Queen reproductive state modulates pheromone production and queen-worker interactions in honeybees. Behav. Ecol. 20 (5), 1007-1014

Lensky, Y., Slabezki, Y. (1981) The inhibiting effect of the queen bee (Apis mellifera L.) foot-print pheromone on the construction of swarming queen cups. J. Insect. Physiol. 27 (5), 313-323

Matsuura, K., Himuro, C., Yokoi, T., Yamamoto, Y., Vargo, E.L., Keller, L. (2010) Identification of a pheromone regulating caste differentiation in termites. Proc. Natl. Acad. Sci. U.S.A. 107, 12963-12968

McDaniel, C.A., Howard, R.W., Blomquist, G.J., Collins, A.M. (1984) Hydrocarbons of the cuticle, sting apparatus, and sting shaft of Apis mellifera L.: identification and preliminary evaluation as chemotaxonomic characters. Sociobiology 8(3), 287-298

Mitchell, C. (1970) Weights of workers and drones. Am. Bee. J. 110, 468-469

Morse, R.A. (1963) Swarm orientation in honeybees. Science 141, 357-358
Naumann, K., Winston, M.L., Slessor, K.N. (1993) Movement of honey bee (Apis mellifera L.) queen mandibular pheromone in populous and unpopulous colonies. J. Insect. Behav. 6(2), 211-223

Niño, E.L., Malka, O., Hefetz, A., Teal, P., Hayes, J., Grozinger, C.M. (2012) Effects of honey bee (Apis mellifera L.) queen insemination volume on worker behavior and physiology. J. Insect. Physiol. 58(8), 1082-1089

Niño, E.L., Malka, O., Hefetz, A., Tarpy, D.R., Grozinger, C.M. (2013) Chemical profiles of two pheromone glands are differentially regulated by distinct mating factors in honey bee queens (Apis mellifera L.). PLoS. One. 8(11), e78637

Peso, M., Niño, E.L., Grozinger, C.M., Barron, A.B... (2013) Effect of honey bee queen mating condition on worker ovary activation. Insectes. Soc. 60 (2), 123-133

Pickett, J.A., Williams, I.H., Martin, A.P., Smith, M.C. (1980) Nasonov pheromone of the honey bee, Apis mellifera L. (Hymenoptera: Apidae) Part I. Chemical Characterization. J. Chem. Ecol. 6(2), 425-434

Pierce, A.L., Lewis, L.A., Schneider, S.S. (2007) The use of the vibration signal and working piping behavior during swarming in honey bees, Apis mellifera. Ethology 113 (3), 267-275

Rangel, J., Seeley, T.D. (2012) Colony fissioning in honey bees: size and significance of the swarm fraction. Insectes. Soc. 59, 453-462

Rangel, J., Reeve, H.K., Seeley, T.D. (2013) Optimal colony fissioning in social insects: testing an inclusive fitness model with honey bees. Insectes. Soc. 60 , 445-452

Richard, F.J., Tarpy, D.R., Grozinger, C.M. (2007) Effects of insemination quantity on honey bee queen physiology. PLoS. One. 2 (10), e980

Richard, F.J., Schal, C., Tarpy, D.R., Grozinger, C.M. (2011) Effects of instrumental insemination and insemination quantity on Dufour's gland chemical profiles and vitellogenin expression in honey bee queens (Apis mellifera ). J. Chem. Ecol. 37 (9), 1027-1036

Schmidt, J.O. (1999) Attractant or pheromone: the case of Nasonov secretion and honeybee swarms. J. Chem. Ecol. 25(9), 2051-2056

Schulz, S., Yildizhan, S., van Loon, J.J.A. (2011) The biosynthesis of hexahydrofarnesylacetone in the butterfly Pieris brassicae. J. Chem Ecol. 37, 360-363

Seeley, T.D. (1979) Queen substance dispersal by messenger workers in honeybee colonies. Behav. Ecol. Sociobiol. 5, 391-415

Seeley, T.D. (1982) Adaptive significance of the age polyethism schedule in honeybee colonies. Behav. Ecol. Sociobiol. 11, 287-293

Seeley, T.D. (2010) Honeybee Democracy. Princeton University Press, Princeton, New Jersey

Seeley, T.D., Buhrman, S.C. (1999) Group decision making in swarms of honey bees. Behav. Ecol. Sociobiol. 45, 19-31 
Seeley, T.D., Fell, R.D. (1981) Queen substance production in honey bee (Apis mellifera) colonies preparing to swarm (Hymenoptera: Apidae). J. Kansas Entomol. Soc. 54 (1), 192-196

Simpson, J. (1963) Queen perception by honeybee swarms. Nature 199, 94-95

Sledge, M.F., Dani, F.R., Fortunato, A., Maschwitz, U., Clarke, S.R., Francescato, E., Hashim, R., Morgan, E.D., Jones, G.R., Turillazzi, S. (1999) Venom induces alarm behaviour in the social wasp Polybioides raphigastra (Hymenoptera: Vespidae): an investigation of alarm behaviour, venom volatiles and sting autotomy. Physiol. Entomol. 24(3), 234-239

Slessor, K.N., Kaminski, L.-A., King, G.G.S., Borden, J., Winston, M.L. (1988) Semiochemical basis of the retinue response to queen honey bees. Nature $\mathbf{3 3 2}$, 354-356

Slessor, K.N., Kaminski, L.A., King, G.G.S., Winston, M.L. (1990) Semiochemicals of the honeybee queen mandibular glands. J. Chem. Ecol. 16, 851-860
Thom, C., Gilley, D.C., Hooper, J., Esch, H.E. (2007) The scent of the waggle dance. PLoS. Biol. 5(9), e228

Winston, M.L., Slessor, K.N., Smirle, M.J., Kandil, A.A. (1982) The influence of a queen-produced substance, 9HDA, on swarm clustering behavior in the honeybee Apis mellifera L. J. Chem. Ecol. 8(10), 1283-1288

Winston, M.L., Slessor, K.N., Willis, L.G., Naumann, K., Higo, H.A., Wyborn, M.H., Kaminski, L.A. (1989) The influence of queen mandibular pheromones on worker attraction to swarm clusters and inhibition of queen rearing in the honey bee (Apis mellifera L.). Insectes. Soc. 36(1), 15-27

Winston, M.L., Higo, H.A., Colley, S.J., Pankiw, T., Slessor, K.N. (1991) The role of queen mandibular pheromone and colony congestion in honey bee (Apis mellifera L.) reproductive swarming (Hymenoptera: Apidae). J. Insect. Behav. 4(5), 649-660

Wyatt, T.D. (2010) Pheromones and signature mixtures: defining species-wide signals and variable cues for identify in both invertebrates and vertebrates. J. Comp. Phys. A. 196, 685-700 